Aus der königl. Universitätsklinil für Hautkrankheiten (Geh.Rat Neisser) und der chemischen Abteilung des königl. physiol. Institutes (Prof. Röhmann) zo. Broslau.

\title{
Was wissen wir über die Zusammensetzung und Entstehung der fettigen Hautsekrete?
}

Von

Dr, C. Sieb̄ert, Bataria, Assistent der Klinik für Hantkrankheiten.

Von Neisser ging die Anregung aus, durch das Studium des Baues und der Funktionen der Bürzeldrüse der Vögel, diesen den menschlichen Talgdrüsen entsprechenden Organen, einigen Aufschluß über die anatomischen und physiologischen Vorgänge bei der Bildung der Hautfette zu gewinnen, und die hierbei gewonnenen Erfahrungen zur Erweiterung unserer Kenntnisse über die Bildung des menschlichen Hautfettes zu benützen. Die erste Frucht dieser Anregung waren Untersuchungen von Plato und Röhmann, die uns sowohl nach mikroskopischer als auch nach physiologisch- chemischer Richtung hin neue interessante Tatsachen lieferten. Von $\mathrm{Röhmann}$ gingen weitere fruchtbringende Anregungen zum Studium des Gebietes der Hautfette aus, an dem sich Linser, Marg. Stern und ich, beteiligt haben.

Die Ergebnisse dieser teils auf chemischem, teils auf mikroskopischem Gebiete sich bewegenden Arbeiten, im Verein mit den wenigen über diese Materie von anderer Seite erfolgten Publikationen, von einem einheitlichen Standpunkte aus zu betrachten und zu erwägen, inwieweit sie das Dunkel, 
das bis jetzt über die Art der physiologischev Funktionen der Talgdrüsen und über die chemische Zusammensetzung des Produktes derselben schwebte, etwas erhellt haben, soll der Zweck der folgenden Abhandlung sein.

Beginnen will ich meine Ausführungen mit den Befunden, die Röhmann bei der genaueren Erforschung der chemischen Zusammensetzung des Bürzeldrüsensekretes erhob. Dieses Sekret müssen wir als ein "Hautfett" resp. "Hauttalg“ auffassen, da es von einem den menschlichen Talgdrüsen in anatomischem Sinne analogen Organe produziert wird, und da es auch demselben physiologisehen Zwecke wie die "Hautfette," nämlich der Einfettung der Körperoberfläche und ihrer epidermoidalen Anhänge dient. $R \ddot{o b m a n n}$ fand bei seinen Untersuchungen zunächst, daß eine der Hauptbestandteile des Bürzeldrüsensekretes, nicht wie de Jonge angenommen hatte, der Cetylalkohol, sondern der Oktadecylalkohol, $\mathrm{C}_{18} \mathrm{H}_{38} \mathrm{O}$, war, also ein Alkohol mit einer hohen Anzahl von Kohlenstoffatomen. Dieser Alkohol ist esterartig gebunden an Stearinsäure, Palmitinsäure und Ölsäure und anscheinend auch an andere kohlenstoffärmere, optisch aktive Säuren. Als einen zweiten wichtigen Befund erhob Röhmann, daß in dem Sekrete nur geringe Mengen von Glyzerin vorhanden waren. Aus diesen beiden Ergebnissen folgte, daß wir das Produkt der Bürzeldrüsen eigentlich nicht als ein "Fett" bezeichnen können, denn Fette in chemischem Sinne sind nur die Glyzerinester der oben genannten Fettsäuren, wie z. B. das Fett des Unterhautfettgewebes. Das Bürzeldrüsensekret reiht sich vielmehr einer anderen Gruppe von chemischen Körpern an, nämlich den "Wachsen“; denn unter "Wachsen" verstehen wir chemische Körper, die aus den Estern der Fettsäuren mit meist einwertigen höheren Alkoholen bestehen, während wir, wie schon erwähnt, in den eigentlichen Fetten Ester von Fettsäuren mit Glyzerin, einem dreiwertigen Alkobol mit nur 3 Kohlenstoffatomen vor uns haben.

Neben den Estern des Oktadecylalkohols fand $\mathrm{Röh} m$ an $n$ in dem Bürzeldrüsensekret noch einen anderen Bestandteil, dessen chemische Natur aber noch nicht vollständig erforsclut ist, den er "Pennacerin" nannte. In dem Pennacerin scheinen wir einen Vertreter einer Gruppe von chemischen Körpern vor 
uns zu haben, die, wie weitere Untersuchungen tierischer und menschlicher Hautfette ergeben haben, durchaus charakteristisch für Produkte tierischer und menschlicher Talgdrüsen zu sein scheint.

Unsere Kenntnisse von dem Sekret der Talg dr ü sen der Säug eti er e beruhte bis vor kurzem wesentlich auf der Untersuchung des Wollfettes der Schafe. Nach den viel zitierten Angaben von Liebreich sollte das Wollfett fast nur aus den Fettsäureestern des Cholesterins $\mathrm{C}_{\mathbf{9} 6} \mathrm{H}_{\mathbf{4 4}} \mathrm{O}$ bestehen. E. Schul z e bestätigte diese Angaben und erweiterte sie dadurch, dab er neben dem Cholesterin noch einen ähnlichen Körper, das Is o cholest er in, auffand. Er machte auch die Beobachtung, daß das Wollfett neben den Estern dieser beiden Alkohole noch andere Stoffe, enthielt, unterwarf diese aber keiner weiteren Prüfung. Daß diese Ester aber nicht immer den Hauptbestandteil des Wollfettes ausmachen, zeigten die Untersuchungen von Darmstädter und Lifschütz. Nach ihren eingehenden Untersuchungen enthält - oder enthielten wenigstens die ron ihnen untersuchten Wollfettproben - nur geringe Mengen jener Ester, sie bestanden aus anderen Verbindungen: "Carnaubasäure, Lanocerinsäure, Lanopalminsäure, Myristinsäure, Carnaubylalkohol, Cerylalkohol." Daß solche Differenzen in dem Ergebnis der chemischen Untersuchung derselben Produkte nicht auf vielleicht verschiedene Untersuchungsmethoden der Autoren zurückzuführen sind, leuchtet ein, und es ist wohl anzunehmen, daß der Grund der verschiedenen Resultate in gewissen Unterschieden des untersuchten Rohmaterials zu suchen sein wird. Über die Momente, die die chemische Zusammensetzung des Wollfettes beeinflussen und derartige Differenzen erklären können, werde ich mich weiter unten auslassen.

Eine Nachprüfung dieser für die Dermatologie äußerst wichtigen Angaben, die ich auf Anregung von $R \ddot{b} h \operatorname{mann}$ ausführte, ergab eine Bestätigung der Angaben von Darmstädter und Lifschütz, insofern als auch ich in dem Wollfett, das mir von den Lanolinwerken Martinikenfelde in dankenswerter Weise zur Verfügung gestellt war, nur geringe Mengen ron Cholesterinestern fand. Ferner ergab sich, daß ein Teil der von Darmstädter und Lifschütz gefundenen Verbindungen 
nicht als solche in dem Wollfett rorhanden sind, sondern erst während der Untersuchung durch Kochen mit alkoholischer Kalilauge entstanden sind. Der Weg der Untersuchung, den ich einschlug, ging darauf aus, die rerschiedenen Bestandteile des Wollfettes möglichst nur durch ihre verschiedene Löslichkeit in Lösungsmitteln, wie Methylalkohol, Äthylalkohol, Äther etc. zu trennen. Es gelang mir verschiedene Körper aus dem Wollfett zu erbalten, von denen der eine bis jetzt am besten charakterisiert, von uns Lanocerin genannt wurde. Aus ihm bildet sich bei der Verseifung anscheinend die von Darmstädter und Lifschütz beschriebene Lanocerinsäure. Mit den weiteren chemischen Untersuchungen des Lanocerins und seiner Spaltungsprodukte ist Röhmann z. Z. noch beschäftigt. In diesem Lanocerin haben wir allem Anscheine nach einen ähnlichen Körper vor uns wie das Pennacerin der Bürzeldrüsensekrete. Das Wollfett scheint also dem Sekret der Bürzeldrüsen insofern zu ähneln, als es wie dieses Ester hochmolekularer Alkohole enthält und andere, wachsartige Stoffe, aber die Alkohole und anscheinend auch die Säuren sind in beiden Sekreten verschieden.

An diese Untersuchungen reihen sich nun weiter die ron Linser über die menschlichen Hautfette. Er gewann normales Hautfett dadurch, daß er Menschen täglich mit einem in Petroläther getränkten Wattebausch abrieb, und dann die von mehreren Wochen aufgesammelten Wattebäusche mit Chloroform extrahierte. Der Rückstand des Chloroformextralktes wurde dann in Äther aufgenommen und der chemischen Untersuchung unterworfen. Nach Verseifung dieser Fettsubstanzen erhielt Linser unter den unverseifbaren Bestandteilen einen Körper, den er Acetonkörper nannte. Cholesterin fand Linser in diesem Hautfett nur etwa $1 \%$, also in verschwindend geringer Menge. Linser untersuchte dann eine Anzahl von anderen menschlichen Fettsekreten, wie $\mathrm{C}$ er umen, $\mathrm{Smegma}$, Inhalt von Talgdrüsencysten, Dermoiden und Atheromen. Bei all diesen Untersuchungen stieß er mit Ausnahme des Cerumens und des Smegmas auf das Yorhandensein des sog. Acetonkörpers. Dieser Körper war kristallinisch und hatte nach mehrmaligem Umkristallisieren einen konstanten Schmelzpunkt 
von 64-68 Grad. Diesen Körper will Rö h man n Dermocerin genannt wissen, da er oder seine Muttersubstanz - der Körper wurde, wie gesagt, erst nach dem Verseifen untersucht - ein dem Pennacerin und Lanocerin analoger Körper zu sein scheint. Aus dem Inhalte von Dermoidcysten gelang es Linser weiter noch einen ölartigen Körper unbekannter Natur zu isolieren, das Dermoolein (Röhmann), das jedenfalls auch kein Fett, kein Alkohol, keine Fettsäure ist. Die nähere Untersuchung dieses Körpers steht auch noch aus. Auch in dem Inhalte von Dermoidcysten, Atheromen etc. fand $\mathrm{L}$ in ser sehr wechselnde und meistens allerdings nur sehr geringe Mengen von Cholesterin. Ebenso verhielten sich auch die Glyzerinfette, so daß hierdurch der Beweis erbracht war, daß wir auch die Produkte der menschlichen Talgdrüsen nicht den eigentlichen Fetten zurechnen können.

Wir haben also aus den Untersuchungen gelernt, daß wir in dem menschlichen Hautfett, dem Wollfett der Schafe, dem Bürzeldrüsensekret der Vögel Produkte vor uns haben, die unter die Gruppe der Wachse zu rubrizieren sind. Vergleichen wir diese Produkte mit den Wachsen der ïbrigen organisierten Welt, so finden wir sowohl nach der chemischen wie nach der funktionellen Richtung hin eine weitgehende Übereinstimmung. Wir sehen, daß eine Ausscheidung von "Wachs" schon auf die Oberfläche von Pflanzen erfolgt. Hier ïberzieht es als eine dünne Schicht Blätter und Früchte, Die Menge des "Pflanzenwachses" ist eine sehr verschiedene; während es bei manchen Früchten, der Weintraube, der Pflaume z. B. nur als ein feiner Hauch erscheint, sondern andere Pflanzen, besonders tropische, soviel Pflanzenwachs $a b$, daß die technische Ausnützung der Wachsproduktion lohnend ist. Von Pflanzen, die so reichlich Wachs bilden, ist besonders die das Carnaubawachs liefernde Carnaubapalme zu nennen, von deren Oberfläche eines Blattes man bis $3 g$ gewinnen kann.

Bei den niederen Tieren ist besonders auffallend die Wachsproduktion bei den Insekten. Das bekannteste Beispiel hierfür ist ja die Honigbiene. Nach Carlet ist das Wachs hier nicht ein Produkt drüsiger Organe, sondern wird von einer epithelialen Membran, der sogenannten Wachsmembran, die 
zwischen der Cuticula und der inneren membranösen Auskleidung der Bauchsegmente gelegen ist, produziert. Im physiologischen Sinne wird man diese Produktion auch als eine Sekretion aufzufassen haben, wenn auch das Produktionsorgan noch nicht zu einer vollendeten Drüse differenziert ist. Weitere Insekten, die wachsartige Produkte liefern, sind Coccus ceriferus, von der das sogenannte chinesische Wachs stammt, ferner die Cochenillelaus, deren Wachs von Liebermann, die Psylla alni, deren Wachs ebenso wie das verschiedener Hummelarten von Sundwick untersucht wurde.

Aus den angeführten Beispielen ersehen wir, daß die Hautoberfläche von Tieren und Pflanzen in einzelnen Fällen diffus, in anderen lokalisiert Produkte ausscheidet, die man zu den Wachsen rechnen kann. Sie bestehen aus den Fettsäure. estern hochmolekularer Alkohole, und anderen Stoffen bzw. Stoffgemischen, die vorläufig als Pennacerin, Lanocerin, Dermocerin (Dermoolein) bezeichnet wurden.

Neben diesen wachsartigen Bestandteilen findet sich in diesen Sekreten noch Cholesterin entweder frei oder als Ester in wechselnden Mengen. Daß wir dem Cholesterin seine Spezifität für Hautsekrete, wie eine solche früher auf Grund der Untersuchungen von Liebreich angenommen wurde, nehmen müssen, lehrten einmal die Untersuchungen des Lanolins, bei dem es nur in einzelnen Handelssorten eine hervorragende Anteilnahme an der Zusammensetzung des Sekretes hat, weiter die Untersuchungen des Bürzeldrüsensekretes, bei dem das Cholesterin überhaupt nicht aufzufinden war, dann die der menschlichen Hautfette, wo es nur eine ganz nebensächliche Rolle spielte.

Es wirft sich nun die Frage auf, wie dieses verschiedene Verhalten des Cholesterins in den Hautfetten, das einmal dominierend auftritt und dann wieder in verschwindend kleiner Menge vorhanden ist, zu deuten ist. Schon liebreich und nach ihm Linser hatten dieses auffallende Verhalten dadurch zu erklären versucht, daß sie annahmen, daß die Hautfette sich aus zwei Teilen zusammensetzen, nämlich einmal aus dem eigentlichen Sekrete der Talgdrüsen und dann aus den Produkten der oberflächlichen Hornschicht der Haut. Letztere Produkte 
sind sehr cholesterinreich, während die der eigentlichen Drüsen cholesterinarm, resp. ganz frei von dieser Substanz sind. Eine Grundlage für diese Annahme gaben Linser die Untersuchungen, die er über den Fettgehalt von Hornsubstanzen angestellt hatte. Er extrahierte Horn- und Hufspäne in Äther, und fand in dem fetthaltigen Extrakt nach der Verseifung eine reichliche Menge von Cholesterin, ganz im Gegensatz zu den Befunden bei den eigentlichen Hautfetten, wo L in s er sowohl in den durch die Chloroformwaschungen gewonnenen Hautfetten, als auch in dem Inhalte von Dermoidcysten nur verhältnismäßig geringe Mengen von Cholesterin fand. Eine Ausnahme nur bildeten die Atherome, deren Inhalt wiederum reichliche Mengen von Cholesterin aufwies, was aber wohl mit der Genese der Atherome, Entstehung aus versprengten epithelialen Keimen und keine Beziehungen zu Talgdrüsen, seinen Grund hatte. Auch in dem makroskopischen Aussehen des Inhaltes der Atherome, der aus mehr hornigen Massen besteht im Gegensatz zu dem mehr fettigen Inhalt der Talgdrüsencysten, zeigt sich die Eigenart seiner Herkunft. Ziehen wir weiter noch das cholesterinfreie Bürzeldrüsensekret in den Kreis unserer Betrachtungen, in dem wir ein vollständig reines Talgdrüsensekret ohne jegliche Beimengung von aus der Epidermis stammenden Bestandteilen haben, so müssen wir Liebreich und Linser recht geben und annehmen, daß sich ein Hautfett, das sehr cholesterinreich ist, aus dem Sekrete der Talgdrüsen und aus Ausscheidungsprodukten der Epidermis zusammensetzt. Der früher erwähnte Wechsel in dem Cholesteringehalte der Wollfette wird nun wohl auch darin zu suchen sein, daß die einzelnen Rohprodukte mehr oder weniger aus der Epidermis stammende Substanzen enthalten werden, was vielleicht mit der mehr oder minder dichten Behaarung der verschiedenen Rassen zusammenhängt.

Auf Grund dieser Tatsache müssen wir also der Epidermis die Fähigkeit zusprechen, Cholesterin-Ester etc. zu bilden, also auch eine gewisse sekretorische Fähigkeit der Epidermis annehmen. Hat diese Annahme nun ihre histologische Basis und läßt dieselbe sich mit dem Mikroskop verfolgen?

Bei dem Fersuche diese Frage zu beantworten, muß ich auf eine Polemik zurückgreifen, die seiner Zeit dadurch hervor- 
gerufen wurde, daß Liebreich die Behauptung autstellte, daß die menschliche Haut Lanolin, $d \mathrm{~h}$. nach der damaligen chemischen Auffassung des Lanolins "Cholesterin-Fette" also Cholesterinester enthalte, und $\mathrm{d} a ß$ das Eleidin und das Keratohyalin, Gemenge von Eiweiß und Cholesterin-Fetten und somit der Ursprung des "Hautlanolins" seien. Lewin, Sticker, Selhorst glaubten mikrochemisch die Hypothese von Liebreich beweisen zu können. Sie bedienten sich zu diesem Zwecke der Liebermannschen Cholestol-Reaktion d. h. Behandlung der Substanzen mit Essigsäure-Anhydrid und konzentrierter Schwefelsäure, einer ursprünglich für Cholesterin angegebenen Reaktion, bei der in vitro eine bestimmte Farbenskala auftritt. Die genannten Autoren ïbertrugen diese Reaktion, wenn auch unter Berücksichtigung gewisser Modifikationen der bei der Reaktion auftretenden Farbenskala, auf die Cholesterinester, und wollten hiermit auch mikrochemisch Cholesterinester in der Epidermis und besonders in der Körnerschicht nachgewiesen haben. Bu z $\mathrm{z}$ ind $\mathrm{S}$ a $\mathrm{n}$ ti konnten die Befunde von $\mathrm{L} \theta$ w in und Sticker im mikrochemischen Sinne nicht bestätigen. Über die auf dieser Cholestolreaktion beruhenden Untersuchungen dieser Polemil können wir kurz hinweggehen, da es nach meiner Meinung gewisse Mißlichkeiten mit sich bringt, wenn man eine Reaktion wie die Cholestolreaktion, die beim Nachweis des Cholesterins sich als so empfindlich erweist, zur Entscheidung über das Vorhandensein oder Fehlen von Cholesterinestern heranzieht. Nehmen wir selbst a priori an, daß die Identifizierung von Cholesterinestern durch die Liebermannsche Reaktion unter Berücksichtigung gewisser Modifikationen in der Farbenskala möglich ist, so liegt doch noch die Möglichkeit vor, daß durch die angewendete konzentrierte Schwefelsäure eine gewisse Spaltung der Ester in ihre Komponenten möglich ist, und daß das frei werdende Cholesterin eine Mischreaktion mit der der Cholesterinfette ergibt, die in gewisser Abhängigkeit von der Menge der angewendeten Schwefelsäure stehen wird. Es kommt dann noch der Umstand hinzu, daß die Produkte, mit denen bei den Untersuchungen die Reaktionen angestellt wurden, sowohl in extrahiertem Zustande als auch in den Geweben bei den mikrochemischen Reaktionen 
nicht reine chemische Körper vorstellen, vou denen man allerdings scharf abgesetzte Farbenreaktionen erwarten darf, sondern daß dieselben doch immer mehr oder weniger Verunreinigungen bekannter und unbekannter Natur enthalten, die die Farbenerscheinungen beeinträchtigen können. Hierzu wird auch der Wassergebalt der Substanzen gehören, der nach $\mathrm{H}$ ïrthle die Farbenerscheinungen der genannten Reaktionen beeinflussen kann. Mir scheint also, daß weder die Untersuchungen von Liebreich, Lewin, Sticker, Selhorst etwas für, noch die von Buzzi und Santi etwas gegen das Vorhandensein von Cholesterinfetten in der Haut beweisen. Durch Anstellung der Reaktion ist nur der Nachweis erbracht worden, daß Cholesterin in der Oberhaut vorhanden ist. Untersuchungen, die direkt gegen die Liebreich sche Behauptung, daß die Körner der Körnerschicht aus Cholesterintetten bestehen, sprechen, sind die, die von $\mathrm{Buzzi}$ über die Löslichkeitsverhältnisse der Körnchen der Körnerschicht angestellt sind. Er fand nämlich, da ) diese in Chloroform, Äther, Essigsäureanhydrid unlöslich sind, und nach der Behandlung mit den oben genannten Fettlösungsmitteln noch gefärbt werden können. Hätte es sich um Cholesterinester gehandelt, so hätten diese leicht in Lösung gehen müssen.

Das Eleidin und das Keratohyalin sind also keine Cholesterinester und nach ihren Löslichkeitsverhältnissen überhaupt keine fettartigen Substanzen. Es ist aber nicht zu leugnen. daß es doch bestimmte Gründe gibt, die dafür sprechen, daß ein gewisser Zusammenhang zwischen Keratohyalin und dem Cholesterin besteht. Vielleicht ist es richtig, was Liebreich auch gelegentlich angedeutet, daß man in dem Keratohyalin gewisse Vorstufen des Cholesterin resp. der Cholesterinester anzusehen hat. Jedenfalls geht das Vorkommen dieser Substanzen, die bekanntlich in einem gewissen Zusammenhang mit den Verhornungsprozessen stehen, im gewissen Sinne parallel. In dem Stratum granulosum findet sich das aus soliden Körnchen bestehende Keratohyalin, das hier noch eiweißartigen Charakter hat (Waldeyer). In den weiter nach oben gelegenen Epithelschichten ändert sich die Substanz derart, daß die Körnchen weicher werden und die Konsistenz eines fetten Öles annehmen, 
Hier haben wir dann das Eleidin oder das Keratoeleidin (Rabl) vor uns. Die einzelnen Tröpfchen konfluieren dann, und erfüllen vollkommen die Zellkörper, die so das Stratum lucidum bilden. Wie sich das Eleidin dann weiter verändert. ist unbekannt, jedenfalls läßt es sich in den verhornten Schichten färberisch nicht mehr nachweisen. Diese verhornten Schichten geben mikrochemisch eine sehr deutliche Cholesterinreaktion. Auch in den ätherischen Extrakten von hornigen Substanzen wie Pferdehufen, Hornspänen, Atherominhalt etc. finden sich wie Linser nachgewiesen hat, reichliche Mengen freien Cholesterins. Von den genannten Hornsubstanzen enthalten Pferdehufe und Hörner auch mikroskopisch viel Eleidin. Als von Bedeutung für den Zusammenhang zwischen Eleidin und Cholesterin könnte man auch noch eine Beobachtung von Dreysel und Oppler heranziehen. Die genannten Autoren fanden in den sehr cholesterinreichen Cholesteatomen reichliche Mengen Eleidin vor. Das Ergebnis dieser Beobachtungen geht also dahin, daß man in der Epidermis dort, wo sich das Eleïdin dem mikroskopischen Nachweise entzieht, Cholesterin deutlich nachweisen kann und daß in sonstigen hornigen Gebilden reichliches Vorhandensein von Eleïdin mit reichlicher Menge von Cholesterin zusammenfällt.

Über die chemische Natur des Eleidins wissen wir weiter nichts Näheres. Wenn auch manche Autoren dazu geneigt sind, diese Substanz als ein fettes Öl anzusehen, so spricht doch die Unlöslichkeit des Eleidins in Fettlösungsmitteln dagegen. Auch über die chemische Qualität des Keratohyalins ist uns nichts Näheres bekannt. Waldeyer will, wie schon erwähnt, die Substanz als einen eiweißartigen Körper aufgefaßt wissen.

Es setzt sich also das fettige Hautsekret der Menschen und der Säugetiere aus zwei Sekreten verschiedener Herkunft zusammen. Ist die Art and Weise, in der der Organismus diese beiden Sekrete aufbaut, nun eine einheitliche? Die Frage, woher das Sekret der eigentlichen Talgdrüsen entsteht, ist durch die Untersuchungen Platos und Röhmanns an der Bürzeldrüse entschieden worden. Plato wies in Versuchen, in denen er Gänse mit Sesamöl fütterte, nach, daß das Sesamöl in 
die Bürzeldrüse eintritt und sogar bis in das Bürzeldrüsensekret gelangen kann. Hiermit war die bisherige Anschauung erschüttert und unwahrscheinlich gemacht, daß sich das Talgdrüsensekret aus dem Eiweiß der Zellen bilde, es sich also um einen fettigen Zerfall der Drüsenepithelien bandle. Die Bildung des Sekretes ist ein regelrechter Sekretionsrorgang. Das der Drüse zugetührte Fett wird aber nicht wie bei der Milchdrüse unverändert ausgeschieden, sondern erleidet zum größten Teil in der Drüse gewisse chemische Veränderungen. Nach Annahme von Röhmann wird das Nabrungsfett in der Drüse fermentativ in Fettsäuren und in Glyzerin gespalten. Ölsäure und Stearinsäure werden zu Oktadecylalkohol reduziert. Kohlenstoffärmere Fettsäuren von der Formel der Myristinsäure und der Laurinsäure führt Röhmann auf einen oxydativen Abbau der Ölsäure, Stearinsäure und Palmitinsäure zurück. Weiter spielen dann noch synthetische Vorgänge eine Rolle, die schließlich zur Bildung des Oktadecylalkoholesters führen. Ferner glaubt Röhmann auch noch Grund zu der Annahme zu haben, daß die Bildung des Pennacerins mit dem Nahrungsfett in einem engen Zusammenhang steht. In der neuesten Zeit ist auch $\mathrm{B}$ ab in einer sehr ausführlichen Arbeit über die Talgdrüsen und ibr Sekret sehr energisch für die Auffassung eingetreten, daß die Fettbildung in der Talgdrüsenzelle ein echter vitaler Sekretionsrorgang ist.

Diese Ausführungen erstrecken sich zunächst nur auf die sekretorischen Vorgänge in den Bürzeldrüsen. Daß wir aber berechtigt sind derartige Verhältnisse auch auf die menschlichen Talgdrüsen zu übertragen, dafür sprechen gewisse Analogien, die sich bei den mikroskopischen Untersuchungen des Sekretionsvorganges zwischen den Bürzeldrüsen und den menschlichen Talgdrüsen ergeben haben und die weiter unten erläutert werden sollen.

Bei dem Hautsekret haben wir noch den cholestearinhaltigen Bestandteil. Über seine Entstehung werden wir uns erst eine Vorstellung bilden können, wenn die Konstitution des Cholesterins endgültig aufgeklärt sein wird.

Während unsere Kenntnisse über die mikroskopischen Vorgänge bei der Bildung des Epidermissekretes noch 
sehr lückenhaft sind und zum größten Teil nur auf Mutmaßungen beruhen, ist unser Wissen über die Vorgänge bei der Fettbildung in der eigentlichen Talgdrüse, besonders in der Bürzeldrüse, durch Plat 0 und Margarete Stern bedentend erweitert worden. Plato fand in dem wabig angelegten Protoplasma der Bürzeldrüsenzellen runde bis ovale, mäßig stark lichtbrechende Körnchen von ziemlich fester Konsistenz, die sich im allgemeinen mit Fettfarbstoffen nicht tingierten. Diese Körnchen nannte Plato lipophore Körnchen, weil er beobachtet haben wollte, daß diese stark lichtbrechenden Gebilde im weiteren Verlauf der Tubuli nach dem Ausführungsgange zu fettartigen Charakter, nachweisbar sowohl durch die Osmium- als auch durch die Scharlachrotreaktion, annahmen, daß sich also gewissermaßen eine fortschreitende Verfettung an diesen lipophoren Körnchen bemerkbar machte. Fortgesetzt wurden die Untersuchungen von M. Stern, welche zunächst durch ihre Untersuchungen nachwies, daß man nicht, wie Plato angenommen, in den lipophoren Körnchen eine Vorstufe des Sekretes, sondern das Selkret selbst vor sich hatte. Außerdem fand M. Stern in den Zellen der Bürzeldrüse noch eine zweite Art von Körnchen, darstellbar durch ein spezifisches Färbeverfahren mittelst Kombination von Osmium und Scharlachrot. Diese Körnchen erhielten den Namen "lipoide" Körnchen. Von den lipophoren Körnchen Platos, welche M. Stern als Sekrettröpfchen bezeicbnete, unterschieden sie sich auch durch geringeres Lösungsvermögen in den Fettlösungsmitteln. M. Ster $\mathrm{n}$ wies in der Bürzeldrüse histologisch drei rerschiedene Zonen, wahrscheinlich funktionell verschiedenen Teilen der Drüse entsprechend, nach. Die lipoiden Körnchen beschränkten sich ausschließlich auf die erste Zone. Die Sekrettröpfchen fanden sich in der ersten und zweiten Zone. In allen drei Zonen kam dann noch eine dritte Art von Körnchen vor, die sich erst nach 9-16 tägiger Einwirkung von Osmiumsäure färbten. Diese Körnchen fanden sich außerdem reihenweise angeordnet in dem intertubulären Bindegewebe und nach neueren Untersuchungen von M. Stern auch in und um die Gefäße, ferner auch in dem! Sekret selbst. 
Die Vorstellung, die uns M. Stern auf Grund dieser Befunde von der Bildung des Bürzeldrüsensekretes gibt, ist folgende: Die staubfeinen Körnchen, die sich in den Zellen und in dem Bindegewebe befinden, sind ein sehr fein verteiltes Nahrungsfett. Ein Teil dieses Nahrungsfettes geht unverändert in das Sekret über, wo es sich auch mikroskopisch nachweisen läßt. Von diesem Nahrungsfett stammen auch die Spuren wirklichen Fettes, die sich in dem Sekrete chemisch nachweisen lassen. Der größere Teil der staubfeinen Körnchen bildet zunäcbst die lipoiden Körnchen, aus denen dann die Sekrettröpfehen (Platos lipophore Körnchen) entstehen. Diese Sekrettröpfchen lassen sich mit ziemlicher Bestimmtheit durch ihr Verhalten zu den Fettlösungsmitteln und durch ihre mangelnde Osmirbarkeit als die Stearin- und Palmitinsäureester des Oktadezylalkohols identifizieren. Auch alle diese Vorgänge weisen wieder mit Bestimmtheit darauf hin, daß die Funktion der Bürzeldrüse eine reine sekretorische ist, und daß das Fett nicht nach der Anschauung von Virchow durch einen nekrobiotischen Prozeß entsteht.

Plato hatte auch menschliche Talgdrüsen in den Bereich seiner histologischen Untersuchungen gezogen und macht hier auf eine wesentliche Übereinstimmung der mikroskopischen Details bei den Talg- und Bürzeldrüsen aufmerksam. Er fand auch in der menschlichen Talgdrüse eine periphere Lage von Zellen, die keine durch Osmium intensir geschwärzte Elemente enthielten, dagegen waren Körnchen zu sehen, welche teils gar keine, teils eine ganz geringe Osmiumbräunung zeigten. Diese Arbeiten wurden durch den Tod des Forschers unterbrochen und M. Stern ist augenblicklich damit beschäftigt, auch diese Untersuchungen noch weiter zu fördern.

Einen ähnlichen Befund, wie Plat o bei den Bürzeldrüsen und bei der menschlichen Talgdrüse erhob auch Buschke beim Studium der Meibo m schen Drüse des Meerschweinchens. Auch hier trat das Sekret zunächst in kleinsten, stark lichtbrechenden Körnchen in den periphersten Punkten in den wohl erhaltenen Zellen auf. Von den Körnchen sagt $B$ u s c h ke, daß dieselben teilweise eine Fettreaktion geben, indem dieselben sich mitunter gar nicht oder mitunter nur partiell mit den Fettfarbstoffen tin- 
gierten. Auch ein Übergehen von Sesamöl in das Sekret dieser Drüsen konnte Buschke in einzelnen Fällen feststellen.

Dieses wäre also der Stand unserer zeitigen chemischen und histologischen Kenntnisse der Hautfette und ibrer Entstehung. Was die physiologische Bedeutung der Hautfette betrifft, so haben die Untersuchungen nach dieser Richtung hin wenig neue Gesichtspunkte geschaffen. Wir sehen den Zweck der fettigen Hautsekrete noch immer als den an, die Körperoberfläche und ihre epidermoidalen Anhänge einzufetten. diese geschmeidig, für Wasser undurchdringlich und nicht benetzbar zu erhalten. Diese Sekrete scheinen dann einen gewissen Schutz gegen bakterielle Einflüsse zu gewähren, insofern als die Hautfette keinen geeigneten Nährboden für Bakterien abgeben.

Gottstein ${ }^{1}$ ) hat experimentell rom Lanolin nachgewiesen, daß in ihm keine Bakterien gedeihen können. Die Erklärung dieser Tatsache liegt in der Natur der Wachse, deren Bestandteile sich als äußerst widerstandsfähig selbst gegen energische chemische Einwirkungen erweisen.

Nach Linser sollen auch die menschlichen Hautfette ebenso wie das Wollfett befähigt sein, gewisse Mengen von Wasser aufzunehmen und hierdurch eine Rolle bei der Wärmeregulation zu spielen, doch erscheint mir diese Annahme nicht ausreichend begründet.

Wir müssen zugeben, daß wir durch die oben angeführten Untersuchungen doch schon um ein Bedeutendes unsere Kenntnisse von den normalen Hautfetten bezuiglich ihrer Chemie und ihrer Entstehung gefördert haben. Andererseits aber ist es noch mit unserem Wissen von der pathologischen Fettsekretion, die für die praktische Dermatologie so wichtige Krankheitsbilder, wie die sogenannte Seborrhoea oleosa und die Seborrhoea sicca hervorruft, schlecht bestellt. Auch wie sich die Fettsekretion bei anderen, außer den genannten Erkrankungen der Haut verhält, ist uns wenig bekannt. Die ersten Anfänge, um in dieses dunkle Gebiet zu dringen, sind auch von Linser gemacht, und diese ersten Untersuchungen haben schon ganz beachtenswerte Resultate geliefert. Was die uns zunächst

1) Berl. klin. Wochenschrift. 1887. 
am meisten interessierenden Krankheiten betrifft, so konnte Linser bei der Seborrhoea sicca und namentlich bei der Seborrhoea oleosa nachweisen, $d a \beta$ das produzierte Hautsekret in einen erheblichen Gegensatz zu dem normalen Hautfett steht. Es zeigie sich, daß diese Sekrete viel reichlicher an freien Fettsäuren waren, als die normalen Hautsekrete, und daß die Vermehrung der freien Fettsäuren hauptsächlich auf Rechnung eines reichen ölsäuregehaltes kam. Linser will daher die Seborrhoea als eine primäre Funktionsanomalie der Talgdrüsen aufgefaßt wissen, infolge welcher eine nicht genügende Verarbeitung des zugeführten Materials, speziell der Ölsäure, stattfindet. Diese Verhältnisse liegen vornehmlich bei der Seborrhoea oleosa vor, bei dem Sekret der Seborrhoea sicca tritt noch ein vermehrter Cholestearingehalt hinzu, der wohl auf eine reichliche Beimengung von Epidermiszellen zum Sekret der Talgdrüsen zurückzuführen ist.

Bei der Untersuchung der Schuppen von Ichthyosiskranken ergab sich ein Resultat, das man nach unseren bisherigen Kenntnissen erwarten durfte, nämlich ein hoher Cholestearingehalt, und ein Zurücktreten der spezifischen Bestandteile der Talgdrüsen. Auch äußerlich dokumentiert sich ja die mangelhafte Funktion der Talgdrüsen bei der Ichthyosis in der ausgesprochenen Trockenheit der Haut. Ein gleiches Resultat, wie die Untersuchung der Ichthyosisschuppen, ergab auch merkwürdigerweise die der Psoriasisschuppen, und Linser macht hier darauf aufmerksam, da $\beta$ anscheinend auch bei der Psoriasis Funktionsstörungen der Talgdrüsen vorkommen könnten.

Diese obigen Ausführungen sind die Resultate der ersten Anfänge, um durch chemisch-physiologische Untersuchungsmethoden in das bis dahin noch so dunkle Gebiet der fettigen Hautsekretion und ihrer Anomalien einzudringen. Es ist zu erwarten, $d a ß$ es durch kombinierte physiologisch-chemische und histologische Untersuchungen gelingen wird, noch weitere Einblicke in diese so dunklen Vorgänge zu gewinnen. 
Zum Schlusse ist es mir eine angenehme Pflicht, Herrn Geheimrat $\mathrm{Neisser}$ für die weitgehende Unterstïtzung bei der Ausführung meiner Untersuchungen und Herrn Professor Röhmann für die Anregungen und die stets bereitwillige Hilfe meinen verbindlichsten Dank auszusprechen.

\section{L i ter a t ur.}

Bab. Die Talgdrüsen u. ihre Sekretion. Beiträge zur klinisch. Mediz. Festschrift Senator.

Buzzi. Monats. f. prakt. Dermat. Bd. VIII. 1889.

Darmstädter u. Lifschütz. Ber. d. Deutsch. chem. Ges. 1898. Bd. XXVIII, ffg.

Dreysel u. Oppler. Archiv für Dermatol. Bd. XXX. 1895.

Hürthle. Zeitschr. f. physiol. Chemie. Bd. XXI. 1895.

de Jonge. Über das Sekret der Talgdrüsen der Vögel und sein Verhältnis zu den fetthaltigen Hautsekreten der Säugetiere, insbesondere der Milch. Inaug.-Diss. Berlin. 1879.

Lewin. Mikrochemischer Nachweis von Cholesterinfett in der Körnerschicht der Epidermis.

Li ebermann. Über das Oxychinoterpen. Ber. d. deutsch. chem. Gesellschaft. 1885 .

Liebreich. Verh. der deutsch. dermat. Gesellschaft u. Berl. klinische Wochenschr. 1885.

Linser. Über den Hauttalg beim Gesunden und bei einigen Hanterkrankungen. Habilitationsschrift. Tübingen. 1904.

Plato. Untersuchangen über die Fettsekretion der Haut. Verhandl. der Deutsch. Dermat. Gesellschaft. Breslau. 1901.

$\mathrm{R}$ öhmann. Über das Sekret der Bürzeldrüsen. Beiträge zur chem. Physiologie u. Pathologie. Bd. V.

- Über das Lanocerin, einen neuen Bestandteil des Wollfettes. Bericht der deutsch. physiolog. Gesellschaft. Zentralblatt für Physiologie. Bd. XIX.

Santi. Monatsh. für prakt. Dermat. Bd. IX. 1889.

Schulze, E. Ber. d. deutsch. chem. Gesellsch. XXXI. Bd. 1898. Schwenkenbecher. Archiv f. klin. Med. Bd. LXXIX. 1904.

Selhorst. Über das Keratohyalin und den Fettgehalt der Haut. Inaug.-Diss. Berlin. 1890.

Stern. Bistologische Beiträge zur Sekretion der Bürzeldrüse. Arch. f. mikrosk. Anatomie u. Entwicklungsgeschichte. Bd. LXVI. 1905.

Sticker. Über die Entwicklung und den Bau des Wollhaares der Schafe. Dissertation. Berlin. 1887.

S undwik. Zeitschrift f. physiol. Chem. Bd. XVII. p. 425.1893. 